\title{
Pattern of osteoarthritis in a West African teaching hospital
}

\author{
A O Adebajo
}

\begin{abstract}
The study of the pattern of osteoarthritis in different populations may yield valuable aetiological clues and also allow subtypes to be defined. Over one year 252 osteoarthritic joints from 140 patients seen at a West African teaching hospital were prospectively reviewed. The knee was the joint most often affected. Hip and hand disease, as well as Heberden's nodes, were uncommon. Joint disease was predominantly monoarticular; no patient had three or more sites affected.
\end{abstract}

The cause of osteoarthritis remains largely unresolved. Although osteoarthritis occurs world wide, both its pattern and prevalence vary among populations.

Information about osteoarthritis is scarce in Africa, including the West African subregion. West Africa is of particular interest as experience with several other rheumatic diseases shows pronounced differences with respect to other areas of the world. ${ }^{1}$ The heterogeneity of osteoarthritis is gaining wider acceptance, and identification of distinct subgroups might provide further information.

\section{Methods}

A prospective study was carried out of all patients with both clinical and radiological features of osteoarthritis attending the rheumatology clinic of the University College Hospital, Ibadan, Nigeria, during the course of one year. Ibadan lies within the Savannah belt of West Africa with an average rainfall of 50 inches and a daily temperature rarely below $20^{\circ} \mathrm{C}$. The teaching hospital serves the city and its environs with a total population of two million. Most patients seen at the hospital are urban dwellers with an urban:rural mix of about $4: 1$.

Medical history was taken and clinical, radiological examination, as well as laboratory investigations were carried out. Age, sex, duration of disease, and occupation were noted, as were predisposing factors in the medical history. Height, weight, and blood pressure were measured in all patients, in addition to an examination of both symptomatic and nonsymptomatic joints. Functional disability was assessed using Steinbrocker's criteria. ${ }^{2}$ Radiographs were limited to symptomatic joints and

Department of Medicine, University College Hospital, Ibadan, Nigeria A O Adebajo

Accepted for publication 11 September 1989 joints showing clinical signs of osteoarthritis. Radiological grading of joints was based on the criteria of Kellgren and Lawrence. ${ }^{3}$ Only patients showing grades 3 and 4 changes by this criteria were included for study. A full haemato- logical profile, erythrocyte sedimentation rate (Westergren), haemoglobin genotype, serum uric acid, urea, electrolytes, and rheumatoid factor determination were performed. Standard methods were used and the latex fixation test was used to screen for rheumatoid factor.

\section{Results}

One hundred and forty patients presenting with 252 affected joints were seen during the study period. One hundred and nine of these patients were female, giving a sex ratio of $1: 3 \cdot 5$. The mean age of onset of disease for these patients was 53.7 (SD 9.0) years with a range of 32-76 years. The median duration of symptoms was one year with a range of two weeks to $\mathbf{4 0}$ years. One hundred and thirteen ( $81 \%$ ) patients gave a history of morning stiffness, the mean duration of which was 24 minutes, with a range of five to 120 minutes.

The sites most commonly affected were the knee, lumbosacral, and cervical spine. The hip, ankle, and wrist joints were least frequently affected (table 1). Excluding the spine, disease in more than one joint invariably included the knee joints with the exception of one patient whose shoulders and elbow joints were affected. No patient had three or more groups of nonaxial joints affected (table 2 ).

Table 1 Distribution of joint disease in 140 osteoarthritic patients

\begin{tabular}{ll}
\hline foint studied & $\begin{array}{l}\text { Number (\%) } \\
\text { of subjects affected }\end{array}$ \\
\hline Knee & $66(47)$ \\
Lumbosacral spine & $60(43)$ \\
Cervical spine & $20(14)$ \\
Hands & $10(7)$ \\
Shoulder & 8 (6) \\
Elbow & 8 (6) \\
Hip & 2 (1) \\
Ankle & 2 (1) \\
Wrist & 2 (1) \\
\hline
\end{tabular}

Table 2 Non-spinal joint distribution by site

\begin{tabular}{lll}
\hline & & $\begin{array}{c}\text { Number of } \\
\text { affected joints }\end{array}$ \\
\hline One joint (n=42): & Knees & 24 (16 right) \\
& Shoulders & 6 (2 right) \\
& Elbows & 6 (2 right) \\
& Hips & 2 (both right) \\
& Anlles & 2 (both right) \\
Wrists & 2 (both left) \\
One site (n=80): & Knees & 66 \\
& Hands & 10 \\
& Shoulder & 2 \\
Two sites (n=5): & Kibow & 2 \\
& Knees/hands & 1 \\
& Knees/hips & 1 \\
& Knees/elbows & 1 \\
& Knoess/ankle & 1 \\
Three or more sites: & Shoulders/elbow & 1 \\
& & 0
\end{tabular}


On the basis of body build, indices for our population as supplied by the dietetic department of the University College Hospital, Ibadan, nine $(6 \cdot 4 \%)$ men and $24(17 \cdot 1 \%)$ women were obese (defined as $10 \%$ above average weight). This compares with $5 \cdot 6 \%$ for men and $10.4 \%$ for women in the normal population. Most of the patients seen worked as market traders or farmers. This pattern of occupation did not differ from that of other clinic attenders; $24.3 \%$ of patients were hypertensive. This compares with a prevalence of $10-16 \%$ in the normal population. ${ }^{4}$ Only two $(1 \cdot 4 \%)$ patients with osteoarthritis had mean diastolic blood pressures over $100 \mathrm{mmHg}$.

Mild to moderate effusions were found in the knees of 13 patients (three men). These knees were not clinically inflamed, neither was there evidence of synovial thickening. In each of these patients with knee effusions the joint was aspirated and the synovial fluid obtained examined for crystals (with the exception of hydroxyapatite crystals). In only two patients were calcium pyrophosphate crystals found. No other joints showed evidence of synovitis and no 'hot' Heberden's nodes were present.

The serum electrolytes, urea, calcium, and phosphate values were all normal for the population. The erythrocyte sedimentation rate (ESR) ranged from 8.0 to $110.0 \mathrm{~mm} /$ first hour with a mean (SD) of $35.6(18.1) \mathrm{mm} / \mathrm{h}$. This is not significantly different from ESR values found in healthy Nigerians. 5

Electrophoretic haemoglobin genotype for sickle cell also showed a normal distribution for the population; 92 patients were AA, $41 \mathrm{AS}$, and seven AC. No patient had either the SS or SC genotype. All patients had normal uric acid concentrations.

Only $3 \%$ of patients were seropositive for rheumatoid factor. Surprisingly, this is lower than the prevalence of $11.5 \%$ found in the sera of healthy Nigerians. ${ }^{1}$

Radiologically the affected joints showed moderate degenerative changes typical of Kellgren's grade 3 . No patients had pronounced or rapidly destructive osteoarthritis. The two patients in whom calcium pyrophosphate crystals were found in the synovial fluid from their knee joints also showed radiographic linear calcification of cartilage. One other patient also showed linear calcification diagnostic of chondrocalcinosis of the knee without an effusion being present. Other joints did not show evidence of chondrocalcinosis. Most knee joints were not pictured in the weight bearing or stressed position as recommended by Ahlback, ${ }^{6}$ but the medial tibiofemoral compartment was most frequently affected radiologically and clinically. The medial tibiofemoral compartment was affected in $65 / 118(55 \%)$ of knee joints, the lateral tibiofemoral compartment in $50 / 118(42 \%)$ of knee joints, and the patellofemoral compartment in $61 / 118(52 \%)$ of knee joints.

Using Steinbrocker's function grading, 110 patients were classified as grade 1, 23 as grade 2, and seven as grade 3 . Surgery was only required for one patient, for whom upper tibial osteotomy was performed.

\section{Discussion}

We found that osteoarthritis was the commonest chronic rheumatic disorder in our clinic over the period under study. With increasing life expectancy as a result of improved health care, one would expect osteoarthritis to become increasingly important in the region. The preponderance of women observed is similar to observations in studies of white populations and contrasts with a preponderance of men found among South African blacks with osteoarthritis. ${ }^{7}$ The slightly earlier age of onset in our patients when compared with subjects in white populations ${ }^{8}$ may reflect the younger mean age of our total population.

Osteoarthritis was found most commonly in the knee joints in our population. A similar observation has been made in Saudi Arabians ${ }^{9}$ and South African blacks. ${ }^{10}$ Most of the patients seen in this study were female traders who sit on low stools about a foot off the ground with their knees in extreme flexion. This position may subject their knee joints to minor trauma and uneven wear. Apart from kneeling for prayers, women are required to kneel as a form of greeting to their elders. The long term effect of this is uncertain. Developmental abnormalities due to Blount's disease ${ }^{11}$ and childhood rickets may also have an important aetiological role. Hypermobility was not a risk factor in this study.

The observed paucity of hip osteoarthritis may be related to the squatting position adopted by the female traders as well as by farmers when sitting on their farms. Similar protection of the hip joint had previously been postulated in the Hong Kong Chinese. ${ }^{5}$ These postulates have not been confirmed, however, and the low incidence of congenital hip abnormalities and dysplasias in West Africa may also play its part. ${ }^{12}$ We did not find any of our patients with the severe osteoarthritic features of Mseleni's disease seen in Southern Africa. ${ }^{13}$ The paucity of Heberden's nodes in our study was striking, as was the low prevalence of multiple joint disease. There is an accompanying paucity of metacarpophalangeal and carpometacarpophalangeal disease in support of the view that such disease is usually linked to multiple joint osteoarthritis. ${ }^{14}$ Most of our patients fit into a category of localised osteoarthritis of the knee. The possibility that this is a distinct subgroup of osteoarthritis warrants further study. Interestingly, our findings support the view that localised osteoarthritis, unlike multiple joint disease, is usually unaccompanied by Heberden's nodes, and increased serum uric acid or rheumatoid factor seropositivity. ${ }^{14}$ The same may also be true of morning stiffness and ESR, but these are difficult to interpret in our population. Determination of the presence and duration of morning stiffness is hampered by difficulties with language interpretation while the ESR is frequently increased in healthy West Africans owing to chronic parasitic infection. ${ }^{1}$ is Our patients with localised knee disease did not show features of an inflammatory osteoarthritis, ${ }^{8}$ and in only three patients were accompanying calcium pyrosphosphate crystals present.

The role of obesity and occupation remains 
unresolved. Unlike the Tswana blacks, ${ }^{7}$ trauma was not a risk factor in our study. Hospital based surveys obviously limit the sample population, and there are undoubtedly people in the community with mild disease. Longitudinal population studies and continued evaluation of osteroarthritis in the region through a rheumatological service are required.

1 Greenwood D M. Polyarthritis in Western Nigeria. I Rheumatoid arthritis. Ann Rheum Dis 1969; 28: 488-96.

2 Steinbrocker O, Traeger C H, Batterman R C. Therapeutic criteria in rheumatoid arthritis. FAMA 1949; 140: 659-62. Kellgren J H, Lawrence J S. Radiological assessment of osteoarthritis. Ann Rheum Dis 1957; 16: 494-502.

4 Johnson T O. Arterial blood pressures and hypertension in an urban African population. British Fournal of Preventive and Social Medicine 1971; 25: 26-33.

5 Hoaglund F T, Yau A C M S, Wong W L. Osteoarthritis of the hip and other joints in Southern Chinese in Hong
Kong-incidence and related factors. I Bone Joint Surg [Am] 1973; 55: 545-7.

6 Ahlback S. Osteoarthritis of the knee. A radiographic study. Acta Radiol (Stockh) 1968; suppl 277: 7-72.

7 Solomon L, Beighton P, Lawrence J S. Osteoarthritis in a rural South African Negro Population. Ann Rhewen Dis 1976; 35: 274-8.

8 Huskisson E C, Dieppe P A, Tucker A K, Cannell L B. Another look at osteoarthritis. Ann Rhewm Dis 1979; 38: 423-8.

9 Rajapakse C N A. The spectrum of rheumatic diseases in Saudi Arabia. Br $\mathcal{O}$ Rheumatol 1987; 26: 22-3.

10 Solomon L, Beighton P, Lawrence J S. Rheumatic disorders in the South African Negro. Part II. Osteoarthritis. S Afr Med F 1975; 49: 1737-40.

11 Golding J S R, McNeil-Smith J D G. Observations on the etiology of tibia vara. $\mathcal{F}$ Bone foint Surg $[B r]$ 1963; 45: $320-5$.

12 Ebong $W W$, Lawson E A L. Pattern of osteoarthritis of the hip in Nigerians. East Afr Med J 1978; 55: 81-4.

13 Wittman W, Fellingham $S A$. Unusual hip disease in remote parts of Zululand. Lancet 1970; i: 842-3.

14 Wood P H N. Osteoarthritis in the community. Clin Rheum Dis 1976; 2: 495-507.

15 Adebajo A O. ARA criteria for rheumatoid arthritis. $\mathrm{Br} \mathrm{J}$ Rheumatol 1989; 28: 177-8. 